\title{
Improved analysis of solar signals for differential reflectivity monitoring
}

\author{
Asko Huuskonen ${ }^{1}$, Mikko Kurri ${ }^{1}$, and Iwan Holleman ${ }^{2}$ \\ ${ }^{1}$ Finnish Meteorological Institute, Helsinki, Finland \\ ${ }^{2}$ Radboud University, Faculty of Science, Nijmegen, the Netherlands \\ Correspondence to: Asko Huuskonen (asko.huuskonen@fmi.fi)
}

Received: 16 February 2016 - Published in Atmos. Meas. Tech. Discuss.: 22 February 2016

Revised: 26 May 2016 - Accepted: 7 June 2016 - Published: 21 July 2016

\begin{abstract}
The method for the daily monitoring of the differential reflectivity bias for polarimetric weather radars is developed further. Improved quality control is applied to the solar signals detected during the operational scanning of the radar, which efficiently removes rain and cluttercontaminated gates occurring in the solar hits. The simultaneous reflectivity data are used as a proxy to determine which data points are to be removed. A number of analysis methods to determine the differential reflectivity bias are compared, and methods based on surface fitting are found superior to simple averaging. A separate fit to the reflectivity of the horizontal and vertical polarization channels is recommended because of stability. Separate fitting also provides, in addition to the differential reflectivity bias, the pointing difference of the polarization channels. Data from the Finnish weather radar network show that the pointing difference is less than $0.02^{\circ}$ and that the differential reflectivity bias is stable and determined to better than $0.04 \mathrm{~dB}$. The results are compared to those from measurements at vertical incidence, which allows us to determine the total differential reflectivity bias including the differential receiver bias and the transmitter bias.
\end{abstract}

\section{Introduction}

Calibration of the radar differential reflectivity $\left(Z_{\mathrm{dr}}\right)$ is crucial for the successful use of dual-polarization measurements (Ryzhkov et al., 2005). For example, a bias of only $0.2 \mathrm{~dB}$ in the differential reflectivity results in $15 \%$ errors on the estimated rain rates (Gourley et al., 2006). Several methods for $Z_{\mathrm{dr}}$ calibration exist which make use of either active (trans- mit and receive) or passive (receive only) observations of unpolarized targets.

The active methods are based on polarimetric properties of rain. Differential reflectivity at vertical incidence is intrinsically zero for raindrops; hence the measured $Z_{\mathrm{dr}}$ is an estimate of the system bias (Gorgucci et al., 1999). A full azimuth rotation is used to improve the estimate. The calibration is also doable at oblique angles using observations of light rain in which the rain drops are closely spherical (Cunningham et al., 2013). Methods using rain observations provide calibration of the full transmitter-receiver chain.

The passive methods are based on using the microwave signals from the sun. The measurements can be off-line measurements, in which the operational scanning is stopped and the radar antenna is pointing at the sun (Pratte and Ferraro, 1989; Melnikov et al., 2003; Ryzhkov et al., 2005; Zrnić et al., 2006) or they can be on-line measurements, in which data from operational scans are used and the normal radar operations need not be stopped (Holleman et al., 2010a; Figueras i Ventura et al., 2012; Frech, 2013; Gabella et al., 2015). Unlike the rain calibration, the solar observations provide information on the receiver chain only, but they provide antenna alignment information in addition.

Holleman et al. (2010a) introduced the on-line method for the solar $Z_{\mathrm{dr}}$ data and showed daily differential reflectivity biases from French and Danish operational polarimetric radars and compared biases to those obtained from rain calibration at zenith. The paper demonstrated the capability and importance of daily monitoring of $Z_{\mathrm{dr}}$. The method builds on those using the solar signals for the antenna pointing and for the monitoring of the radar receiver chain stability (Darlington et al., 2003; 
Huuskonen and Hohti, 2004; Holleman and Beekhuis, 2004; Huuskonen and Holleman, 2007; Holleman et al., 2010b). Figueras i Ventura et al. (2012) studied one year of data from French radars and concluded that both the solar method and the zenith calibration fluctuate less than $\pm 0.2 \mathrm{~dB}$ and that the fluctuations stem mainly from the variability of the receiver chain. Frech (2013) showed results on antenna pointing and received power and determined the pointing difference of the two channels by analysing the data from horizontal and vertical channels separately, finding a value of about $0.02^{\circ}$. Cunningham et al. (2013) show results on the operational WSR-88 network. Gabella et al. (2015) describe a method for estimating the $Z_{\mathrm{dr}}$ bias by the difference of the 11th largest $\mathrm{H}$ and $\mathrm{V}$ reflectivity values.

In the present paper, we revisit the monitoring of the receiver chain of the $Z_{\mathrm{dr}}$ calibration by the on-line solar method. We discuss the filtering of the raw solar hit data to remove rain and clutter contamination with the aim of increasing the quality and number of the solar hits. We then present several ways to obtain the $Z_{\mathrm{dr}}$ bias and discuss their usability. We also study the two polarization data sets separately, which produces results on the pointing difference between the polarizations. Results are compared with those obtained from the $Z_{\mathrm{dr}}$ calibration in rain with zenith pointing antenna.

\section{Data}

The Finnish Meteorological Institute (FMI) operates a network of $10 \mathrm{C}$-band Doppler weather radars, of which nine radars are polarimetric. Every $15 \mathrm{~min}$ the radars perform a 12-elevation volume scan between 0.3 and $45^{\circ}$ elevations, where 6 elevations up to $9^{\circ}$ are scanned in single-PRF with $570 \mathrm{~Hz}$, and then 6 elevations starting from 2 up to $45^{\circ}$ in dual-PRF. Every $5 \mathrm{~min}$ the first 6 of these 12 elevations are repeated. A description of the FMI network is given by Saltikoff et al. (2010). Two new radars have been added to the network since then, and the network upgrade to polarimetry has continued so that all radars except one are polarimetric. For convenience, some relevant properties of the five radars used in this study are shown in Table 1.

\section{Method}

\subsection{Detection of sun signatures}

The detection of solar signatures in polar volume data of weather radars is described in Huuskonen and Holleman (2007). In the method a reflectivity signal which originates from a continuous microwave source is searched along radials in the operational scan data. Figure 1 shows an example where four radars observe the solar signal simultaneously close to the spring equinox. As a radar signal processor usually corrects the received echoes for the range de-

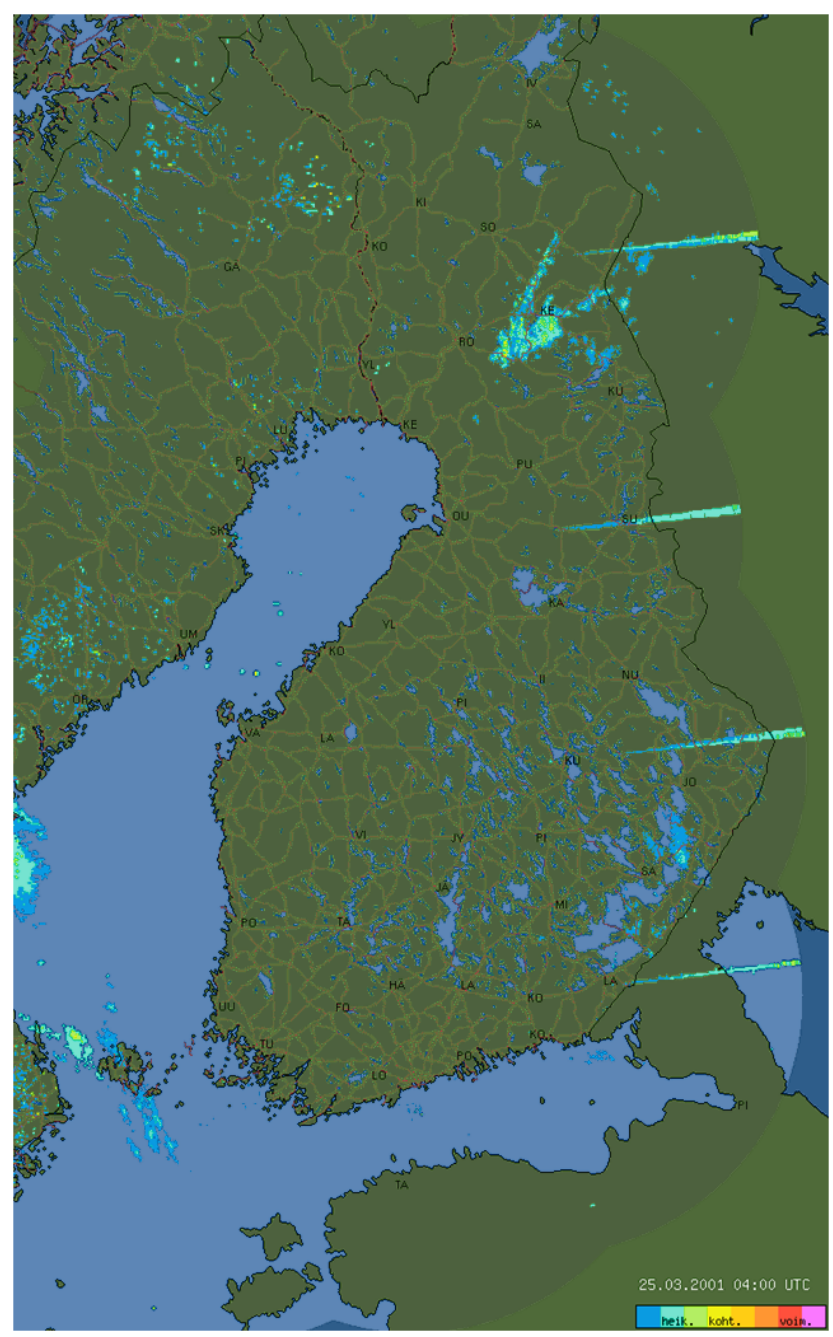

Figure 1. Solar signals observed simultaneously by four radars in Finland on 25 March 2001 at 04:00 UTC.

pendence and the atmospheric attenuation, the "reflectivity" signals from the sun increase as a function of range. The received solar spectral power at the antenna feed $P_{\mathrm{H}, \mathrm{V}}$ for the horizontal and vertical polarizations (per $\mathrm{MHz}$ in $\mathrm{dBm}$ ) can be calculated from the reflectivity signature as a function of range $Z_{\mathrm{H}, \mathrm{V}}(r)$ in $\mathrm{dBZ}$ :

$$
P_{\mathrm{H}, \mathrm{V}}=Z_{\mathrm{H}, \mathrm{V}}(r)-20 \log _{10} r-2 a r-C_{\mathrm{H}, \mathrm{V}}-10 \log _{10} \Delta f,
$$

where $C_{\mathrm{H}, \mathrm{V}}$ is the radar constant in $\mathrm{dB}$ according to ProbertJones (1962) for horizontal and vertical polarizations respectively, $a$ is the one-way gaseous attenuation in $\mathrm{dB} \mathrm{km}^{-1}$, and $\Delta f$ is the receiver $3 \mathrm{~dB}$ bandwidth in $\mathrm{MHz}$, assumed to be the same for both polarization channels. In the case of a proper solar signal, the power $P$ is constant along the range. Depending on the hardware of the radar, the volume coverage pattern, the season, and the latitude of the radar, several tens of sun hits are found per day. Uncorrected reflectivity data (i.e. noise subtracted but ground clutter filtering not applied) 
Table 1. Some properties of the FMI radars relevant to this study. The columns give the radar name, three letter acronym, latitude and longitude of the radar, the antenna beam widths for the horizontal polarization $(\mathrm{H})$ and vertical polarization $(\mathrm{V})$ in elevation and azimuth directions in degrees, the difference of losses in the transmitter chain $\left(T_{x}\right)$ in $\mathrm{dB}$, and difference of the antenna gains $(g)$ in dB. The beam widths in the elevation and azimuth directions are based on measurement of the electric $(\boldsymbol{E})$ and magnetic fields $(\boldsymbol{H})$ as indicated in parentheses.

\begin{tabular}{lcccccccccc}
\hline Radar & Code & lat $^{\circ} \mathrm{N}$ & lon $^{\circ} \mathrm{E}$ & $\mathrm{H}(\mathrm{el}, \boldsymbol{H})$ & $\mathrm{H}(\mathrm{az}, \boldsymbol{E})$ & $\mathrm{V}(\mathrm{el}, \boldsymbol{E})$ & $\mathrm{V}(\mathrm{az}, \boldsymbol{H})$ & $T_{x, h}-T_{x, v}$ & $g_{h}-g_{v}$ \\
\hline Korpo & KOR & 60.13 & 21.64 & 0.914 & 0.980 & 0.940 & 0.941 & 0.0 & -0.1 \\
Anjalankoski & ANJ & 60.90 & 27.11 & 0.909 & 0.974 & 0.967 & 0.923 & 0.0 & 0.0 \\
Kesälahti & KES & 61.91 & 29.80 & 0.927 & 0.949 & 0.944 & 0.928 & 0.0 & -0.2 \\
Utajärvi & UTA & 64.77 & 26.32 & 0.911 & 0.960 & 0.930 & 0.903 & -0.3 & 0.0 \\
Luosto & LUO & 67.14 & 26.90 & 0.911 & 0.983 & 0.953 & 0.943 & -0.2 & 0.0 \\
\hline
\end{tabular}

are used for this analysis, as especially time-domain Doppler clutter filters can attenuate the solar signal by several dBs. The solar signal may also have contamination caused by ground clutter and precipitation. This can be circumvented by discarding data below $1^{\circ}$ elevation and using data from long ranges (e.g. $>100 \mathrm{~km}$ ) only.

\subsection{Modelling of $Z$ signatures}

The sun hits have a symmetric distribution around the sun position which is slightly wider in azimuth than in elevation. A typical example is shown in the left panel of Fig. 2. The larger width in azimuth is caused by the integration while the antenna is scanning, but is also influenced by the averaging window (Zrnić and Doviak, 1976; Huuskonen et al., 2014a). The distribution of linear powers is well approximated by a Gaussian form; hence the power $P_{\mathrm{H}, \mathrm{V}}$ for the horizontal and vertical polarizations in $\mathrm{dB}$ can be written as follows:

$P_{\mathrm{H}, \mathrm{V}}(x, y) \equiv a_{x} \cdot x^{2}+a_{y} \cdot y^{2}+b_{x} \cdot x+b_{y} \cdot y+c$,

where the coordinates $x$ and $y$ are defined as follows:

$x=\left(\phi_{\text {read }}-\phi_{\text {sun }}\right) \cdot \cos \theta_{\text {sun }}$

$y=\theta_{\text {read }}-\theta_{\text {sun }}$,

where $\phi$ and $\theta$ denote azimuth and elevation, "read" refers to the angle reading of the radar antenna, and "sun" refers to the calculated sun position. The observed azimuth differences are multiplied by $\cos \theta_{\text {sun }}$ to make them invariant to the elevation (e.g. Doviak and Zrnić, 1993, p. 516). Equation (2) is linear in the parameters $a_{x}$ to $c$, and thus the sun data can easily be fitted to this equation by the least squares method. Parameters $a_{x}$ and $a_{y}$ are related to the widths of the distributions of the $x$ and $y$ values, parameters $b_{x}$ and $b_{y}$ to the elevation and azimuth biases, and parameter $c$ to the peak power, i.e. when the antenna is pointing exactly at the sun. Note that these parameters need to be defined separately for the horizontal and vertical polarizations. We assume that the biases and widths are independent of the pointing angles, and that the microwave centre of the sun is close to the centre of the optical disk. The elevation width $\Delta_{\theta}$, the azimuth width $\Delta_{\phi}$, the elevation bias $B_{\theta}$, the azimuth bias $B_{\phi}$, and the power when the antenna is pointing directly to the sun, $\hat{P}_{\text {sun }}$, can be calculated from the linear parameters (Huuskonen and Holleman, 2007):

$\Delta_{\phi, \theta}^{2}=-\frac{40 \log _{10} 2}{a_{x, y}} \approx-\frac{12}{a_{x, y}}$

$B_{\phi, \theta}=-\frac{b_{x, y}}{2 a_{x, y}}$

$\hat{P}_{\mathrm{H}, \mathrm{V}}=c-\frac{b_{x}^{2}}{4 a_{x}}-\frac{b_{y}^{2}}{4 a_{y}}$.

The widths are obtained from Eq. (5) when the corresponding parameter $a_{x, y}$ is negative.

As data from different elevations are analysed together, the solar elevation needs to be corrected for the effects of refraction. We use the analytical formulas for the atmospheric refraction of radiowaves, which are consistent with the commonly used $k$-model or 4/3-model (Holleman and Huuskonen, 2013). As the solar signal traverses all atmosphere, the expected value of $k$ is less than $4 / 3$, which is valid close to the surface. Hence we use $k=5 / 4$, which fits best to the model calculations and radar observations according to Holleman and Huuskonen (2013). To avoid severe refraction, data below $1^{\circ}$ elevation are discarded.

\subsection{Modelling of $Z_{\mathrm{dr}}$ signatures}

The modelling of the $Z_{\mathrm{dr}}$ signatures depends on which quantities are calculated in the radar signal processor. In case the horizontal reflectivity factor $Z_{\mathrm{H}}$ and the vertical reflectivity factor $Z_{\mathrm{V}}$ are both available separately, the analysis described above is repeated for both polarizations. An estimate of the solar $Z_{\mathrm{dr}}$ is obtained from the difference of the horizontal and vertical solar powers, $P_{\mathrm{H}}-P_{\mathrm{V}}$, by applying Eq. (1):

$Z_{\mathrm{dr}} \equiv Z_{\mathrm{H}}-Z_{\mathrm{V}}=P_{\mathrm{H}}-P_{\mathrm{V}}+\left(C_{\mathrm{H}}-C_{\mathrm{V}}\right)$.

In addition one obtains the pointing difference of the horizontal and vertical polarizations, and it is also possible to determine how the widths compare to each other.

If the quantities available from the signal processor are $Z_{\mathrm{H}}$ and $Z_{\mathrm{dr}}$, the vertical reflectivity can be calculated as 

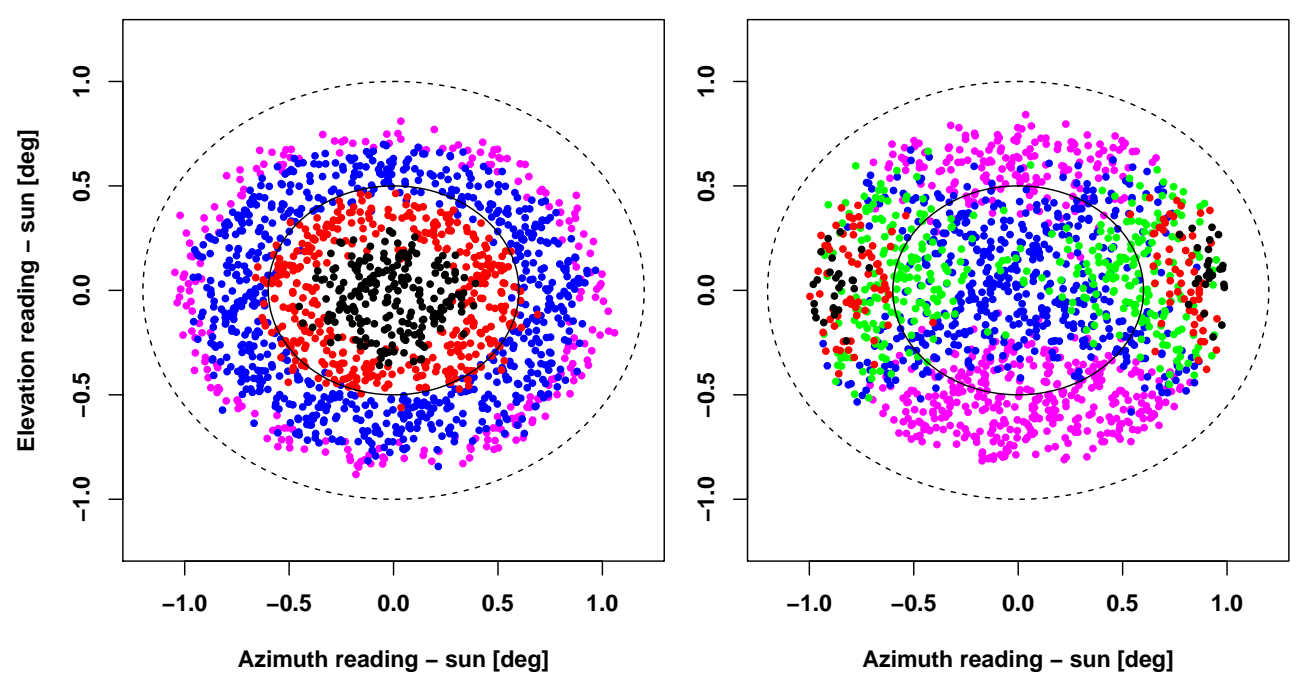

Figure 2. Sun images based on sun hits collected from the FMI Anjalankoski radar in March 2015. The left panel shows the sun hit power relative to maximum (black within $1 \mathrm{~dB}$ of maximum, red $1 \ldots 3 \mathrm{~dB}$ below the maximum, blue $3 \ldots 7 \mathrm{~dB}$ below the maximum, and magenta more than $7 \mathrm{~dB}$ below the maximum) and the right panel the differential reflectivity (magenta less than $-0.3 \mathrm{~dB}$, blue $-0.3 \ldots-0.1 \mathrm{~dB}$, green $-0.1 \ldots 0.1 \mathrm{~dB}$, red $0.1 \ldots 0.3 \mathrm{~dB}$, and black above $0.3 \mathrm{~dB}$ ). An ellipse with axes of 1 and $1.2^{\circ}$ is provided to show the approximate half power $(3 \mathrm{~dB})$ widths in elevation and azimuth respectively. The dashed lines show ellipses with axes lengths twice the size.

$Z_{\mathrm{H}}-Z_{\mathrm{dr}}$, and the procedure outlined above can be followed. In the FMI system the signal processor uses the same radar constant for both polarizations. Then, in fact, the quantity provided by the signal processor is the difference of powers $P_{\mathrm{dr}}=P_{\mathrm{H}}-P_{\mathrm{V}}$ instead of $Z_{\mathrm{dr}}$ calculated from the reflectivity factors. Also in this case the analysis can be carried out as above, using the same radar constant for $H$ and $V$ processing when applying Eq. (1). The $C_{\mathrm{H}}-C_{\mathrm{V}}$ factor is in this case included in the system $Z_{\mathrm{dr}}$ bias, and taken into account when the system bias is subtracted (see Sect. 4.3).

It is also possible to do the analysis directly to $Z_{\mathrm{dr}}$, following the procedure of Holleman et al. (2010a). If we expand Eq. (8) using the expressions for the horizontal and vertical powers in Eq. (2) and equal the coefficients of the resulting equation with those of the two-dimensional polynomial for $\hat{Z}_{\mathrm{dr}}$, which is

$Z_{\mathrm{dr}} \equiv \bar{a}_{x} \cdot x^{2}+\bar{a}_{y} \cdot y^{2}+\bar{b}_{x} \cdot x+\bar{b}_{y} \cdot y+\bar{c}$

and apply Eqs. (5) to (7), we arrive at the following equations:

$$
\begin{aligned}
\bar{a}_{x} & =a_{x, h}-a_{x, v} \approx-12 \cdot\left(\frac{1}{\Delta_{\phi, h}^{2}}-\frac{1}{\Delta_{\phi, v}^{2}}\right) \\
\bar{a}_{y} & =a_{y, h}-a_{y, v} \approx-12 \cdot\left(\frac{1}{\Delta_{\theta, h}^{2}}-\frac{1}{\Delta_{\theta, v}^{2}}\right) \\
\bar{b}_{x} & =b_{x, h}-b_{x, v}=-2 \cdot\left(a_{x, h} B_{\phi, h}-a_{x, v} B_{\phi, v}\right) \\
& \equiv-2 \bar{a}_{x} \bar{B}_{\phi} \\
\bar{b}_{y} & =b_{y, h}-b_{y, v}=-2 \cdot\left(a_{y, h} B_{\theta, h}-a_{y, v} B_{\theta, v}\right) \\
& \equiv-2 \bar{a}_{y} \bar{B}_{\theta}
\end{aligned}
$$

$\hat{Z}_{\mathrm{dr}}=\bar{c}-\frac{\bar{b}_{x}^{2}}{4 \bar{a}_{x}}-\frac{\bar{b}_{y}^{2}}{4 \bar{a}_{y}}$,

where $\bar{B}_{\phi}$ is the azimuth bias, $\bar{B}_{\theta}$ is the elevation bias, and $\hat{Z}_{\mathrm{dr}}$ is the differential reflectivity at the extreme point. The curvature equations, (Eqs. 10) and (11), reveal that the curvatures in elevation and azimuth may have the same or opposite signs. Hence the $Z_{\mathrm{dr}}$ surface is either an elliptic or a hyperbolic, i.e. a saddle surface. The pointing equations (Eqs. (12) and (13)) indicate that whenever the $\mathrm{H}$ and $\mathrm{V}$ pointing directions agree, $Z_{\mathrm{dr}}$ is also pointing to the same direction. This is easily seen, e.g. if Eq. (12) is solved for the bias:

$\bar{B}_{\phi}=\frac{a_{x, h} B_{\phi, h}-a_{x, v} B_{\phi, v}}{a_{x, h}-a_{x, v}}$.

When the curvatures (i.e. widths) of the horizontal and vertical polarizations agree, either in azimuth or elevation, the $Z_{\mathrm{dr}}$ curvature is zero, and the pointing is undefined. Expansion of the right hand side of Eq. (14) shows that, when a pointing difference between the two polarizations is present, $\hat{Z}_{\mathrm{dr}}$ does not evaluate to $Z_{\mathrm{H}}-Z_{\mathrm{V}}$ as defined in Eg. (8) but there are in addition terms proportional to the pointing difference. Hence the direct fitting to $Z_{\mathrm{dr}}$ implies that the pointing differences are assumed to be small.

The right panel of Fig. 2 shows a scatter plot of the differential reflectivity, based on the same data as the reflectivity in the left panel. The distribution looks very different from that of the reflectivity, as the curvature has opposite signs in the elevation and azimuth directions. The distribution has the form of a saddle surface. In the vertical (elevation) axis the values are negative at the edges indicating that the $\mathrm{V}$ lobe 
is wider than the $\mathrm{H}$ lobe, whereas in the azimuth direction the opposite is true, in agreement with the antenna measurements shown in Table 1 and Eqs. (10) and (11). The saddle surface form is different from the symmetric form seen in the Trappes radar data (Holleman et al., 2010a). In the Trappes case the surface has a minimum in the solar direction, indicating that the horizontal beam is wider than the vertical beam in all directions.

\subsection{Calibration of $Z_{\mathrm{dr}}$ using zenith scans}

Calibration of differential reflectivity using polarimetric properties of rain has first been demonstrated by Gorgucci et al. (1999). Differential reflectivity at vertical incidence is intrinsically zero for raindrops; hence the measured $Z_{\mathrm{dr}}$ is an estimate of the system bias $Z_{\mathrm{dr}}^{\text {bias }}$, which is a sum of the differential biases in reception, $\Delta R_{\mathrm{dr}}$, and transmission, $\Delta T_{\mathrm{dr}}$ :

$Z_{\mathrm{dr}}^{\mathrm{bias}}=\Delta R_{\mathrm{dr}}+\Delta T_{\mathrm{dr}}$.

Here $\Delta R_{\mathrm{dr}}$ and $\Delta T_{\mathrm{dr}}$ consist of differences of waveguide and other losses and the antenna gain between the horizontal and vertical polarization channels, and $\Delta T_{\mathrm{dr}}$ is in addition affected by the differences in the transmitted power between the channels.

For $Z_{\mathrm{dr}}$ bias estimation, all FMI polarimetric radars scan $360^{\circ}$ in azimuth with vertically pointing antenna every $15 \mathrm{~min}$. A full azimuth scan is performed to reduce the effects of the orientation of the scatterers or possible asymmetries caused by the radome and the water on it. There is a transient feature during the first few kilometres from the radar, because the horizontal and vertical receiver channels (i.e. the $T / R$ limiters) return to their normal operation in a different way after the transmission pulse. The range of this effect is radar dependent and varies from 2 to $7 \mathrm{~km}$ for the FMI radars. Because of transient effect, $Z_{\mathrm{dr}}$ bias is analysed from altitudes where the transient has died out. Figure 3 shows an example of the $Z_{\mathrm{dr}}$ bias analysis. In the analysis an average of $Z_{\mathrm{dr}}$ values of the sweep over $360^{\circ}$ in azimuth is calculated for each altitude level defined by the $125 \mathrm{~m}$ range gate used in these measurements. As a quality measure, only data with cross correlation coefficient $\rho_{\mathrm{HV}}>0.9$ and SNR $>5 \mathrm{~dB}$ are accepted for the analysis, and data beyond two standard deviations from the mean are discarded. If more than $80 \%$ of the $360^{\circ}$ azimuth sector is covered by good quality data points, the mean $Z_{\mathrm{dr}}$ bias is calculated for that altitude level. This last requirement is used to mitigate the potential $Z_{\mathrm{dr}}$ bias caused by the orientation symmetry of the scatterers or by other disturbances during the measurement. This procedure is repeated for every vertical scan, and the final result is calculated as a daily mean of these quality controlled measurements.

The climatological melting layer height in Finland during the summer is about $3 \mathrm{~km}$, which means that most of the data used for the analysis are obtained from solid precipitation. Snow is not spherical, but the assumption of zero intrinsic

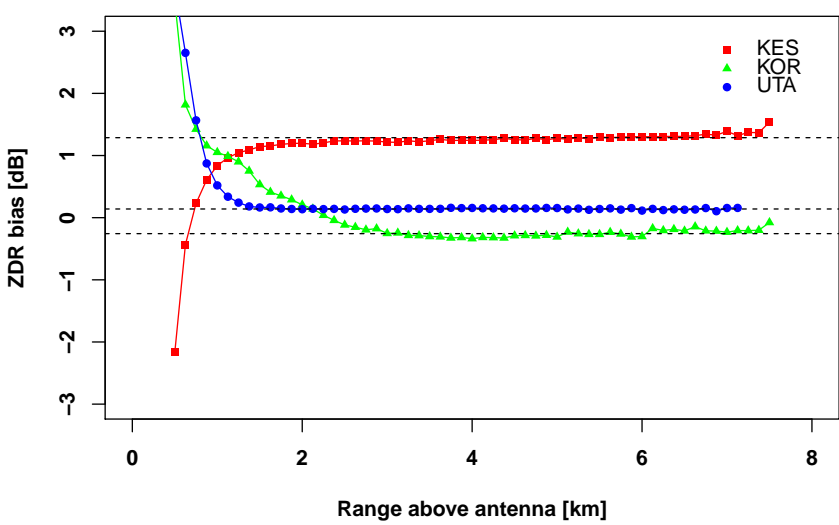

Figure 3. $Z_{\mathrm{dr}}$ as a function of altitude above the antenna estimated from zenith measurements during precipitation events on 6 June 2015. Each point is a daily average of measurements from a full azimuth rotation of the antenna. The dashed lines indicate the $Z_{\mathrm{dr}}$ bias calculated as explained in the text.

$Z_{\mathrm{dr}}$ is justified because of the random motion of the snow, and the averaging in the azimuth. For the case in Fig. 3 the upper edge of the melting layer is at about $2 \mathrm{~km}$ altitude. All curves are continuous and smooth across that altitude.

\section{Results and discussion}

\subsection{Quality control of the solar hits}

The quality control of the solar hit data is necessary to get results of good quality. Huuskonen and Holleman (2007), Holleman et al. (2010b) and Huuskonen et al. (2014a) used a two-stage approach for ensuring that rain and clutter do not contaminate the hit data. The first stage is to use data from far ranges only, e.g. $100 \mathrm{~km}$ (Huuskonen et al., 2014a), which guarantees that clutter is not included, and the hit average is made of points above the rain in most cases. In addition standard selection methods, such as removing data points with high standard deviations, were applied. The second stage was to do the fitting of data to the model twice. After the first fit, data points too far (e.g. $1 \mathrm{~dB}$ ) from the fitted curve are removed and a second fit is carried out on the remaining points. This method works efficiently when a small number of outliers appears in the data, unless they are strong or far from the solar direction so that the first fit is too far from the truth. The results are further improved if one assumes that the antenna is already well pointed and uses only hits close to that direction. This is an efficient method for avoiding using signal from RLANs (Radio Local Area Network), which produce signatures resembling those of the sun. The use of these methods guarantees that good results are obtained in a majority of cases. As the antenna pointing or the calibration levels are not adjusted on a daily basis based on these results, occasional bad results cause no trouble. Altube et al. (2015) describe a partially similar set of methods which offer the 


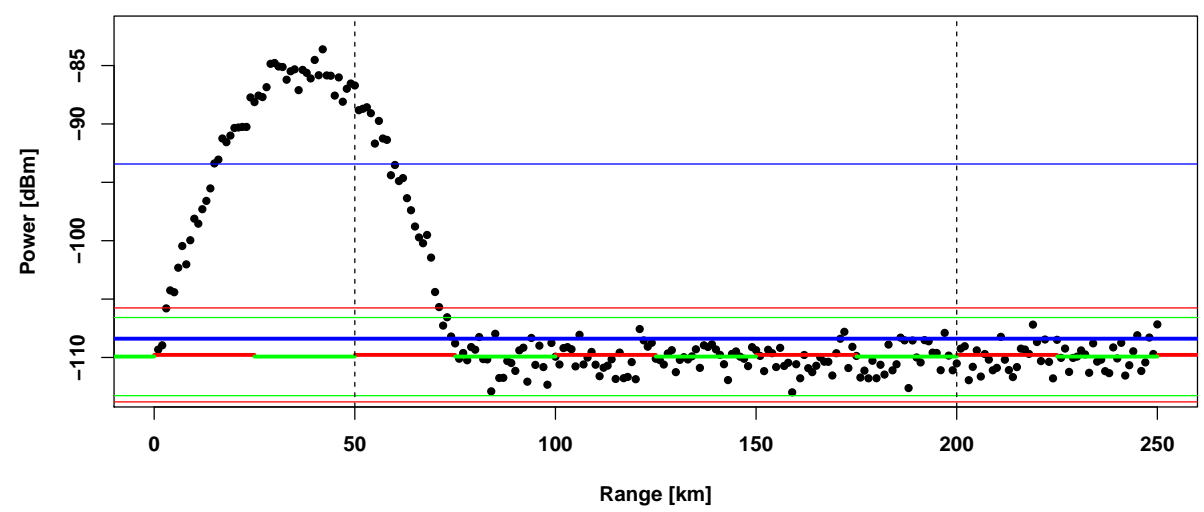

Figure 4. Statistical estimators in simulated rain with noise. The thick lines give three estimators of the centre point: mean from $50 \mathrm{~km}$ distance (blue), median from $50 \mathrm{~km}$ distance (red), mean from $200 \mathrm{~km}$ distance (green). The thin lines of the respective colours indicate the filtering windows. The second blue thin line at $-123 \mathrm{dBm}$ is not shown. The window width for the mean estimator is 3 times the standard deviation and for the median estimator is 3 times the median absolute deviation scaled by 1.4280 so that it agrees with the standard deviation of the underlying normal distribution. The two range limits are indicated by vertical dashed lines.

same functionality for the improvements of the quality of solar hits.

The number of sun hits and the statistical accuracy can be increased by also using data from closer ranges. Then the probability that the data are contaminated by rain or clutter increases, and one has to devise a method for removing the contaminated data prior to calculating the solar hit power. One possible method is a two-stage estimation, in which the first estimate of the solar hit power is calculated from the full data set, and this power together with an estimate of the width of the distribution is used to remove non-solar data. This is illustrated in Fig. 4, which shows a simulated solar hit case with rain at ranges less than $75 \mathrm{~km}$. The figure shows that the mean of data between the $50 \mathrm{~km}$ and the $250 \mathrm{~km}$ ranges (blue) is much higher than the median of the same data (red), which in turn is close to the mean of data beyond the $200 \mathrm{~km}$ range (green). If the last is taken as an unbiased estimator of the solar power, the conclusion is that the median is a much better estimator than the mean when all data beyond $50 \mathrm{~km}$ is used. The median represents the solar power as long as clearly more than half of the points are genuine solar hit points, but will of course be more biased when the amount of contaminated points increases. Evidently, the power estimate based on far ranges only is even better but less precise due to lower number of samples used and less robust against outliers. The estimates of the width of the distribution confirm the above. If the standard deviation is used to determine the width of the distribution, the estimate is biased by precipitation contamination, as indicated in the figure. The median absolute deviation (MAD) gives a width estimate which is close to the width estimated from far ranges only.

Figure 5 shows the result of applying the methods to 1 month of solar hit data from the Anjalankoski radar. Instead of fixed range limits, as in Fig. 4, altitude limits are used, which enables us to use data from several elevation angles

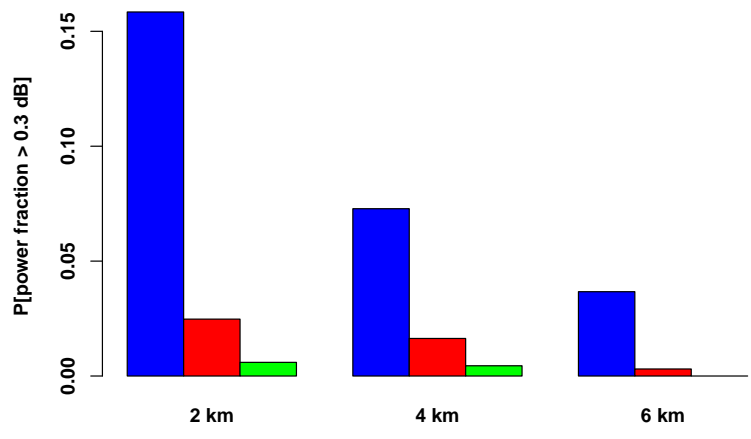

Figure 5. Probability that the solar hit power exceeds the far range power by $0.3 \mathrm{~dB}$. The power determined by mean filtering is denoted by blue, and the power determined by median filtering by red. The green bar indicates solar power determined by the mean filtering when the filtering window is determined from points above $8 \mathrm{~km}$ altitude. The probability is given for start altitudes of 2,4 , and $6 \mathrm{~km}$.

together. The figure illustrates the probability that any of the estimators produce a biased estimate of the solar hit power. The reference power is obtained by calculating the mean hit power above $8 \mathrm{~km}$, which is free of rain contamination for the data used in the study. Figure 5 shows that filtering by mean produces biased estimates even if the start altitude is put to $6 \mathrm{~km}$, and that the number of biased estimates is significant for lower start altitudes.

The overall best performance is obtained if the filtering window is first estimated using data from high altitudes and the final estimate of the mean is calculated using data also from the lower altitude. Then a biased estimate is obtained only in very few cases. The median estimator, although nearly as good, produces biased estimates of the power in some cases. One can reduce the computational load for realtime analysis of large data sets, e.g. for the analysis of all 
European solar hit data within the OPERA (Operational Programme for Exchange of Weather Radar Information) data centre (Huuskonen et al., 2014b) by fixing the width of the filtering window instead of estimating it for each solar hit. A recommended value is at least 3 times the standard distribution deviation of the solar hit data, amounting in the FMI case to about $2 \mathrm{~dB}$. Fixing the window width is possible because the statistics of the solar hit data do not vary from case to case, as pointed out by Altube et al. (2015).

The effect of the improved filtering is also noticeable in the fitted parameters. For the case in Fig. 5, the number of good solar hits increases by about $10 \%$ and the root mean square error of the fit decreases by about $3 \%$ when the two better filtering methods are used instead of the simple mean filtering (blue bar). This holds when the data are used starting from $2 \mathrm{~km}$ upwards. The improvement is case dependent and depends to a great extent on the number of rainy days in the data set. In the analysis we have discarded all solar hits with standard deviation greater than $2.5 \mathrm{~dB}$, which is about 3-4 times the standard deviation of genuine solar hits. Without this additional screening, the improvement when using the two better methods would be much greater.

The filtering of the differential reflectivity $Z_{\mathrm{dr}}$ is not as straightforward as the filtering of the reflectivity, because $Z_{\mathrm{dr}}$ in rain and from the sun do not deviate significantly from each other. The data contaminated by rain can be removed prior to averaging by using the reflectivity data as a proxy to indicate which data points are based on sun and which on rain. Hence the estimates for $Z_{\mathrm{dr}}$ are calculated as the mean and standard deviation of the $Z_{\mathrm{dr}}$ profile by applying the same range indices as had been used for the reflectivity data.

\section{2 $\quad Z_{\mathrm{dr}}$ results based on solar hits}

There is a number of different methods to estimate the $Z_{\mathrm{dr}}$ bias from the solar hits. Holleman et al. (2010a), when solving Eq. (9) for the $Z_{\mathrm{dr}}$, first estimated the widths using a larger data set and fixed the widths in the fitting to improve the stability of the fit. This is one of several methods available for the estimation of the $Z_{\mathrm{dr}}$ bias. An obvious second choice is to do a full 5-parameter fit, such as recommended for reflectivity data (Huuskonen et al., 2014a; Altube et al., 2015). It is also possible to do 3-parameter fitting by fixing the pointing to that obtained from the reflectivity fit, and thus fitting for the power and the two width values only. Noting that the $Z_{\mathrm{dr}}$ bias would be constant over the solar disk for fully matching antenna beams, one can take a mean or a median of all $Z_{\mathrm{dr}}$ hits. And, finally, it is possible to analyse the reflectivity channels separately, either by a 5- or 3-parameter fit, and get the estimate by differencing the power.

Figure 6 compares $Z_{\mathrm{dr}}$ estimates by five methods presented above for 1 month of data. In the following we assess the methods by their ability to estimate correctly the value of $Z_{\mathrm{dr}}$ at direct solar pointing. One can readily notice that the

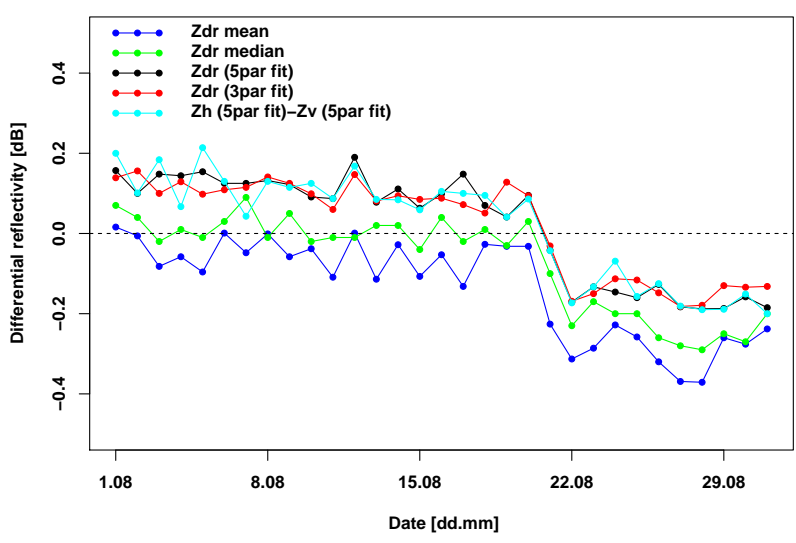

Figure 6. Solar $Z_{\mathrm{dr}}$ values with system $Z_{\mathrm{dr}}$ bias subtraction for the ANJ radar in August 2014. The methods are indicated in the figure. The downward step on 21 August is a result of the change of the system $Z_{\mathrm{dr}}$ bias.
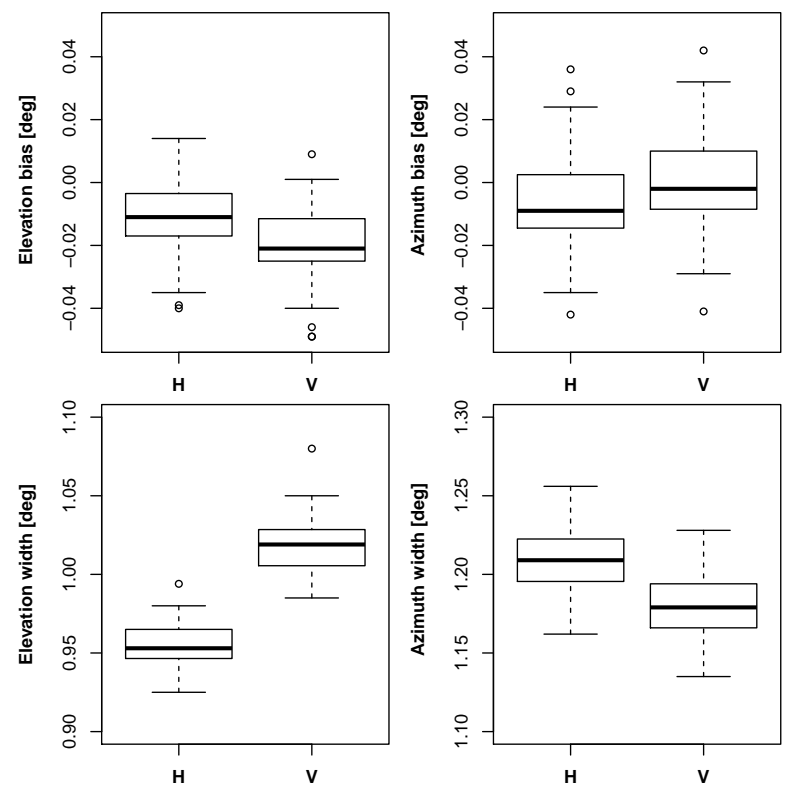

Figure 7. Elevation and azimuth pointing and image width results for $\mathrm{H}$ and $\mathrm{V}$ polarizations for 1 month of data of the ANJ radar. In each panel, the median is shown with a thick line at 1st and 3rd quartiles by a box. Whiskers indicate data points closer than 1.5 times the box length from the quartiles, and data points beyond that are marked with circles.

results obtained by using the mean or the median are clearly different from those obtained by the fitting methods. This is not surprising, because the $Z_{\mathrm{dr}}$ field seen in Fig. 2 is not constant. Taking a mean might work for a saddle surface if the averaging is restricted to a small area around the solar direction, but would not work if the $Z_{\mathrm{dr}}$ surface has a clear minimum or maximum at the solar direction, such as for the case shown in Holleman et al. (2010a). Hence these methods are not recommendable. 

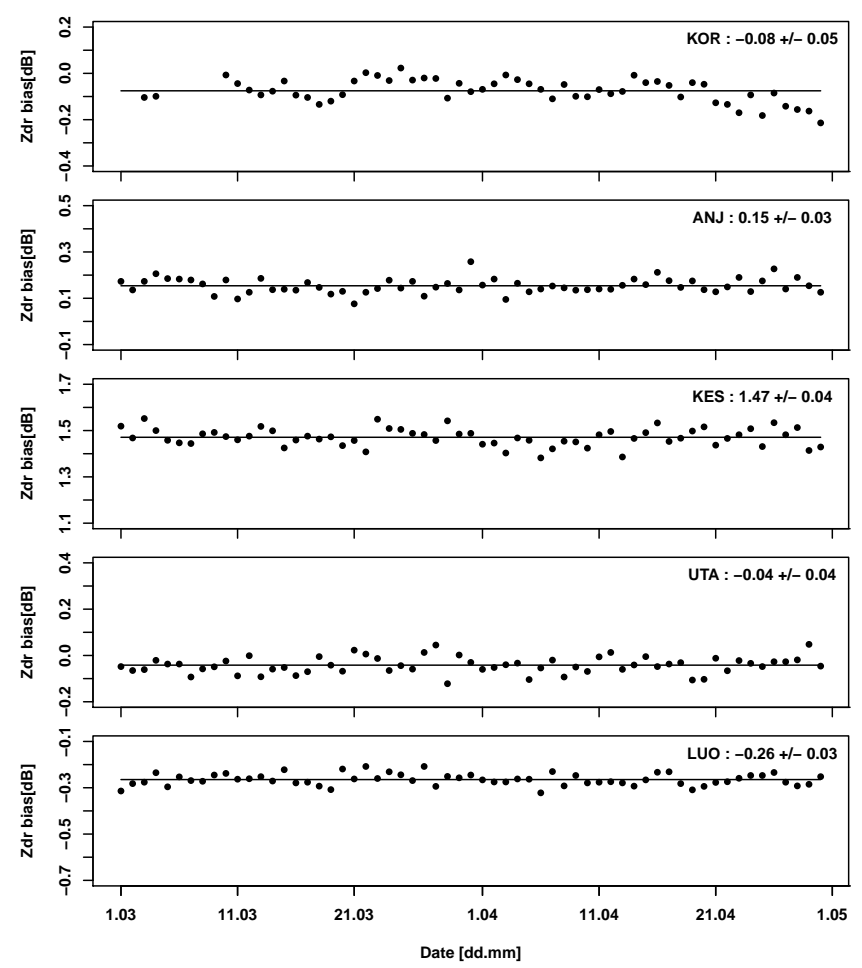

Figure 8. $Z_{\mathrm{dr}}$ results from solar signals for March and April 2015 for FMI radars indicated in the panels. The system $Z_{\mathrm{dr}}$ bias has not been subtracted from the data. The numbers in each panel give the mean and standard deviation for the data.

The three fitting methods give comparable results in the FMI case, where the widths of the two polarizations differ. If the azimuth and elevation widths are close to each other, the direct fitting to $Z_{\mathrm{dr}}$ becomes more unstable, because the errors of the power and the widths are correlated. And if widths in either elevation or azimuth (or both) agree, the surface degenerates into a plane in that dimension, and the fitting to $Z_{\mathrm{dr}}$ is ill posed as an inverse problem and not at all possible. Hence a direct fit to the $Z_{\mathrm{dr}}$ is not guaranteed to work for all radars. A separate 5-par fit to both $P_{\mathrm{H}}$ and $P_{\mathrm{V}}$ is instead a safe method which works in all cases, and therefore recommended. The $Z_{\mathrm{dr}}$ result is then obtained as the difference of the determined powers. If the solar SNR is low, a 5-par fit may not converge. One can then improve the stability of the fitting by fixing the widths to theoretical values or values based on large amount of data, as recommended by Huuskonen and Holleman (2007).

The additional benefit of doing the fitting to both polarization channels separately is that one can determine the angle between the pointing directions of the two polarizations. The two upper panels of Fig. 7 show statistics of the elevation and azimuth pointing results of the $\mathrm{H}$ and $\mathrm{V}$ polarizations for 1 month of data for the Anjalankoski (ANJ) radar. The analysis confirms that the $\mathrm{H}$ and $\mathrm{V}$ beams are well aligned. The pointing difference between $Z_{\mathrm{H}}$ and $Z_{\mathrm{V}}$ is less than $0.02^{\circ}$
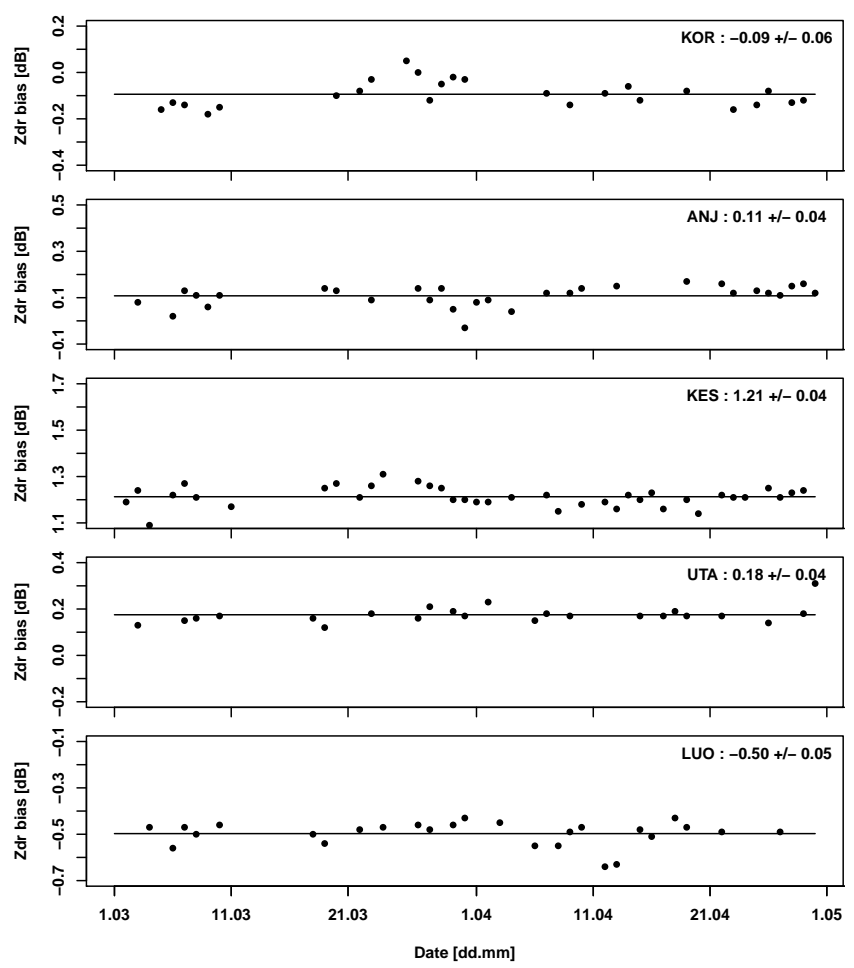

Figure 9. $Z_{\mathrm{dr}}$ results in rainfall from the zenith scan for March and April 2015 for FMI radars indicated in the panels. The numbers in each panel give the mean and standard deviation for the data. For each radar the same vertical axis limits as in Fig. 8 are used.

for ANJ. A similar analysis of all the radars confirms that for most other radars in the network the pointing difference is less than $0.01^{\circ}$. These pointing angle differences are similar to those reported earlier by Frech (2013) for the German network.

The lower panels compare the width values of the two polarizations. There is a clear difference in the image widths so that for the $\mathrm{V}$ polarization the image is wider in elevation and for the $\mathrm{H}$ polarization in the azimuth. This is a result of the antenna design, and the results are typical for the whole network and are consistent with the width values given in Table 1.

Figure 8 shows $Z_{\mathrm{dr}}$ results from five polarimetric FMI radars, based on separate 5-par fitting to $P_{\mathrm{H}}$ and $P_{\mathrm{V}}$, as recommended above. As the measured quantities in the FMI system are $Z_{\mathrm{H}}$ and $Z_{\mathrm{dr}}, Z_{\mathrm{V}}$ has been calculated as their difference. The $Z_{\mathrm{dr}}$ results are differences of the fitted horizontal and vertical powers, without subtracting the system $Z_{\mathrm{dr}}$ bias. Figure 8 shows that the bias varies from radar to radar and that the standard deviation ranges from 0.03 to $0.05 \mathrm{~dB}$. This is an indication of the stability of the radar system itself and of the analysis method. The standard deviations are significantly lower than the $0.2 \mathrm{~dB}$ reported by Holleman et al. (2010a), probably due to the better rain rejection in the estimation of the hits. The standard deviations are also some- 
what lower than the $0.05 \mathrm{~dB}$ reported by Gabella et al. (2015), which were obtained as differences of median power values instead of fitted powers.

\section{3 $Z_{\mathrm{dr}}$ results based on zenith scans in precipitation}

Figure 9 shows $Z_{\mathrm{dr}}$ bias values based on the zenith scan measurements for the same radars as used in Fig. 8. The results are not available for all days, because the analysis requires precipitation above the radar. Yet the bias has been determined for more than half of the days. It is seen that the $Z_{\mathrm{dr}}$ bias varies from radar to radar but that the bias is stable as indicated by the standard deviation of the data. Comparison with Fig. 8 shows that standard deviations are slightly higher than those for the solar data.

Both the solar and the zenith methods are based on using an object with intrinsic zero differential reflectivity. Yet the results are not directly comparable, because the solar results depend on the receiver biases only, whereas the zenith results depend both on the receiver and the transmitter sides. One could, in principle, use the known loss and gain biases to correct the observations. Any significant deviation of the corrected value from zero would indicate an error in the loss and gain figures. In systems where both polarizations are processed using a single radar constant, one needs to include the $C_{\mathrm{H}}-C_{\mathrm{V}}$ factor, discussed in Sect. 3.3, in the estimation.

In many operational systems, the zenith $Z_{\mathrm{dr}}$ is used as the system bias which is subtracted from all $Z_{\mathrm{dr}}$ measurements, including the solar ones. The system bias is a sum of transmitter and receiver biases (Eq. 16); hence the receiver biases cancel out and the result should amount to $-\Delta T_{\mathrm{dr}}$, when the system bias is subtracted. The possibly existing $C_{\mathrm{H}}-C_{\mathrm{V}}$ factor also cancels out in the operation. Comparison of the sum of the differential transmitter losses and antenna gains given in Table 1 with the difference of the solar measurements in Fig. 8 and zenith measurements in Fig. 9 shows that the two estimates are within $0.1 \mathrm{~dB}$ for four radars and about $0.5 \mathrm{~dB}$ for one radar. Noting the stability of the results from both the solar and zenith methods and assuming that the antenna gains do not change over time, the most obvious explanation is that the transmitter losses are not correct. The fact that the transmitter loss values might be incorrect does not affect the accuracy and usability of the $Z_{\mathrm{dr}}$ measurements in precipitation, because the zenith scan measurement takes all loss factors into account, and the bias determined from it corrects for all possible errors in the loss or gain difference values.

The interpretation of the solar $Z_{\mathrm{dr}}$ measurements depends on how often the system bias is updated. Usually the system bias is updated manually once the zenith observations indicate that the existing value is no more valid. In this case the solar method is useful for monitoring the receiver side, even though the solar $Z_{\mathrm{dr}}$ with system bias subtraction amounts to $-\Delta T_{\mathrm{dr}}$. Clearly, if something changes in the receiver chain, the solar $Z_{\mathrm{dr}}$ changes readily. If something changes in the transmitter chain, excluding the antenna, no effect is seen in the solar $Z_{\mathrm{dr}}$ until the system bias is updated. The effect of such a change in the system bias is seen in Fig. 6 in which the $Z_{\mathrm{dr}}$ values make a downward step on 21 August. If the system bias was updated in near real time on a daily basis, the solar $Z_{\mathrm{dr}}$ would actually follow the changes on the transmitter side. Daily setting of the system bias is not usual, and not even possible, because it requires the presence of precipitation.

\section{Conclusions}

The differential reflectivity of the quiet sun is zero and constant over the solar surface but the radar measurements also include the effect of the antenna and the receiver chain. In case the beam shapes of the horizontal and vertical polarizations were fully identical, all $Z_{\mathrm{dr}}$ observations would have a constant value over the solar surface. This is not the case as shown by examples in Holleman et al. (2010a) and in the present paper. Hence the $Z_{\mathrm{dr}}$ bias cannot be estimated accurately by taking a simple median or mean over all solar $Z_{\mathrm{dr}}$ observations. Instead it is necessary to fit the observations to a model. The most stable and recommended method is to make a full 5-parameter fit to both polarization channels separately, which works well also when the $Z_{\mathrm{dr}}$ surface approaches constant, which corresponds to zero curvature, i.e. matching width values between polarizations. In that case direct fitting to $Z_{\mathrm{dr}}$ would become increasingly difficult. In case the widths of the two polarizations differ, it is also possible to perform a 3-par or 5-par fit to $Z_{\mathrm{dr}}$ directly. The FMI radar software provides the horizontal reflectivity $Z_{\mathrm{H}}$ and the differential reflectivity $Z_{\mathrm{dr}}$, and the estimated vertical reflectivity $Z_{\mathrm{V}}$ is not fully calibrated. We obtain a true estimate of the solar $Z_{\mathrm{dr}}$ also in this case, but a radar software which provides both $Z_{\mathrm{H}}$ and $Z_{\mathrm{V}}$ simplifies the analysis and is recommended for use.

The zenith measurements of $Z_{\mathrm{dr}}$ in precipitation include both the transmitter and the receiver chain, whereas the solar measurements include the receiver chain only. The zenith measurements are essential because they are used to estimate the $Z_{\mathrm{dr}}$ bias, which is subtracted in the signal processing from all $Z_{\mathrm{dr}}$ measurements. The solar $Z_{\mathrm{dr}}$ observations are valuable for the monitoring of the receiver stability, and they also provide a consistency check of the transmitter losses and gains. If the solar $Z_{\mathrm{dr}}$ bias is not close to $-\Delta T_{\mathrm{dr}}$ after application of the zenith bias correction, the transmitter losses and gains are suspect. For the monitoring, the solar observations are most valuable, because an estimate is obtained during most days, unlike the zenith observations which are obtained only during precipitation.

The statistical accuracy of the $Z_{\mathrm{dr}}$ results, both solar and zenith observations, is better than $0.04 \mathrm{~dB}$ for most radars, based on the analysis of 1 month of data. This is a better value than those reported earlier. The accuracy is a combination of the radar system performance and that of the analysis sys- 
tem. In the latter we have used a number of existing methods (Huuskonen and Holleman, 2007; Holleman et al., 2010a) and introduced a number of new tools. With this we have developed a method to extract solar hits accurately without any significant rain and clutter contamination. Noting that the $Z_{\mathrm{dr}}$ from the sun and the signals we want to remove, e.g. clutter and rain, may be close to each other in value, we have introduced a method where the quality control is done using the reflectivity data. The improved level of accuracy provided by these methods allows us to monitor and detect changes in the receiver chain much better than before. All this improves the quality of $Z_{\mathrm{dr}}$ data, which is most important for many polarimetric algorithms. It is recommended that this improved online monitoring of the differential reflectivity is performed daily for all polarimetric radars in the network, preferably in combination with the rain calibration at zenith.

Acknowledgements. The numerical analysis and the figures have been prepared using the R software (R Core Team, 2015).

Edited by: G. Vulpiani

\section{References}

Altube, P., Bech, J., Argemí, O., and Rigo, T.: Quality control of antenna alignment and receiver calibration using the Sun: adaptation to mid range weather radar observations at low elevation angles, J. Atmos. Ocean. Technol., 32, 927-942, 2015.

Cunningham, J., Zittel, W., Lee, R., Ice, R., and Hoban, N.: Methods for identifying systematic differential reflectivity $\left(\mathrm{Z}_{\mathrm{DR}}\right)$ biases on the operational WSR-88D network, in: 36th Conference on Radar Meteorology, AMS, 16-20 September 2013.

Darlington, T., Kitchen, M., Sugier, J., and de Rohan-Truba, J.: Automated Real-time Monitoring of Radar Sensitivity and Antenna Pointing Accuracy, in: 31st Conference on Radar Meteorology, 538-541, AMS, 5-12 August 2003.

Doviak, R. J. and Zrnić, D. S.: Doppler Radar and Weather Observations, Academic Press, Second edn., 562 pp., ISBN-10: 0-48645060-0, 1993.

Figueras i Ventura, J., Boumahmoud, A.-A., Fradon, R., Dupuy, P., and Tabary, P.: Long-term monitoring of French polarimetric radar data quality and evaluation of several polarimetric quantitative precipitation estimators in ideal conditions for operational implementation at C-band, Q. J. Roy. Meteor. Soc., 138, 22122228, 2012.

Frech, M.: Monitoring the data quality of the new polarimetric weather radar network of the German Meteorological Service, in: 36th Conference on Radar Meteorology, AMS, 16-20 September 2013.

Gabella, M., Sartori, M., Boscacci, M., and Germann, U.: Vertical and Horizontal Polarization Observations of Slowly Varying Solar Emissions from Operational Swiss Weather Radars, Atmosphere, 6, 50-59, 2015.

Gorgucci, E., Scarchilli, G., and Chandrasekar, V.: A Procedure to Calibrate Multiparameter Weather Radar Using Properties of the
Rain Medium, IEEE Trans. Geosci. Remote S., 37, 269-276, 1999.

Gourley, J. J., Tabary, P., and Parent du Chatelet, J.: Data quality of the Meteo-France C-band polarimetric radar, J. Atmos. Ocean. Technol., 23, 1340-1356, 2006.

Holleman, I. and Beekhuis, H.: Weather Radar Monitoring using the Sun, Technical Report TR-272, Royal Netherlands Meteorological Institute (KNMI), 2004.

Holleman, I. and Huuskonen, A.: Analytical formulas for refraction of radiowaves from exoatmospheric sources, Radio Science, 48, 226-231, doi:10.1002/rds.20030, 2013.

Holleman, I., Huuskonen, A., Gill, R., and Tabary, P.: Operational Monitoring of Radar Differential Reflectivity using the Sun, J. Atmos. Ocean. Technol., 27, 881-887, 2010a.

Holleman, I., Huuskonen, A., Kurri, M., and Beekhuis, H.: Operational Monitoring of Weather Radar Receiving Chain using the Sun, J. Atmos. Ocean. Technol., 27, 159-166, 2010 b.

Huuskonen, A. and Hohti, H.: Using the solar flux data from operational scans for checking the elevation pointing of a radar, in: 3rd European Conference of Radar Meteorology and Hydrology (ERAD), 6-10 September 2004.

Huuskonen, A. and Holleman, I.: Determining weather radar antenna pointing using signals detected from the sun at low antenna elevations, J. Atmos. Ocean. Technol., 24, 476-483, 2007.

Huuskonen, A., Kurri, M., Hohti, H., Beekhuis, H., Leijnse, H., and Holleman, I.: Radar performance monitoring using the angular width of the solar image, J. Atmos. Ocean. Technol., 31, 17041712, 2014a.

Huuskonen, A., Saltikoff, E., and Holleman, I.: The operational weather radar network in Europe, Bull. Am. Meteorol. Soc., 95, 897-907, 2014b.

Melnikov, V. M., Zrnić, D. S., Doviak, R. J., and Carter, J. K.: Calibration and Performance Analysis of NSSL's Polarimetric WSR-88D, Tech. rep., National Severe Storms Laboratory (NSSL), available at: http://www.nssl.noaa.gov/publications/ wsr88d_reports/Calibration_and_Performance_Analysis.pdf (last access: 11 July 2016), 2003.

Pratte, J. F. and Ferraro, D. G.: Automated solar gain calibration, in: 24th Conference on Radar Meteorology, 619-622, AMS, 27-31 March 1989.

Probert-Jones, J. R.: The radar equation in meteorology, Q. J. Roy. Meteor. Soc., 88, 486-495, 1962.

R Core Team: R: A Language and Environment for Statistical Computing, R Foundation for Statistical Computing, Vienna, Austria, available at: https://www.R-project.org/ (last access: 11 July 2016), 2015.

Ryzhkov, A. V., Giangrande, S. E., Melnikov, V. M., and Schuur, T. J.: Calibration issues of dual-polarization radar measurements, J. Atmos. Ocean. Technol., 22, 1138-1155, 2005.

Saltikoff, E., Huuskonen, A., Hohti, H., Koistinen, J., and Järvinen, H.: Quality Assurance in the FMI Doppler Radar Network, Boreal Env. Res., 15, 579-594, 2010.

Zrnić, D. S. and Doviak, R.: Effective antenna pattern of scanning radars, IEEE Trans. Aer. Electronic Systems, 12, 551-555, 1976.

Zrnić, D. S., Melnikov, V. M., and Carter, J. K.: Calibrating Differential Reflectivity on the WSR-88D, J. Atmos. Ocean. Technol., 23, 944-951, 2006. 